\title{
Estratégia Multidimensional na Redução de Infeção Associada a Cateter Venoso Central em Pediatria
}

\author{
Multidimensional Strategy Regarding the Reduction of \\ Central-Line Associated Infection in Pediatric Intensive Care
}

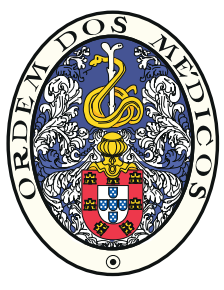

\author{
Jorge RODRIGUES ${ }^{1}$, Andrea DIAS $\square^{1,2}$, Guiomar OLIVEIRA ${ }^{1,3}$, José Farela NEVES ${ }^{1,2}$
}

Acta Med Port 2016 Jun;29(6):373-380 - http://dx.doi.org/10.20344/amp.5558

\section{RESUMO}

Introdução: Determinar a incidência de infeções da corrente sanguínea associadas ao uso de cateter venoso central, após reforço de medidas multidisciplinares de boa prática e a sua comparação com a taxa de incidência de infeções da corrente sanguínea associadas ao uso de cateter venoso central prévia.

Material e Métodos: Estudo observacional descritivo, com colheita prospetiva de dados, durante cinco meses, após implementação de medidas multidisciplinares. Foram incluídas todas as crianças admitidas na unidade de Cuidados Intensivos Pediátricos, submetidas à colocação de cateter venoso central e foi efetuada comparação com controlos históricos.

Resultados: Incluíram-se 75 doentes com idade mediana de 23 meses: 22 (29,3\%) recém-nascidos, 28 (37,3\%) submetidos a cirurgia e $32(43,8 \%)$ com patologia subjacente. Foram colocados 105 cateteres venosos centrais, com tempo médio de permanência de 6,8 $\pm 6,7$ dias. O tipo de cateter venoso central mais comum foi de curta duração $(45,7 \%)$, sendo os locais de inserção mais frequentes a veia subclávia e da flexura braquial (ambos $25,7 \%$ ). Não ocorreu nenhuma infeção da corrente sanguínea associada ao uso de cateter venoso central durante o período do estudo. Comparando com os controlos históricos, ambos os grupos eram semelhantes relativamente à idade, género, proveniência dos doentes e local de colocação de cateter venoso central. No estudo atual, a duração mediana de internamento foi superior, com tempo de permanência de cateter venoso central (excluindo epicutâneo-cava) semelhante. Não se verificou diferença em relação ao calibre e número de lumens do cateter venoso central utilizado. A percentagem de crianças que colocou cateter venoso central em relação ao total de crianças admitidas no serviço no mesmo período foi menor no estudo atual, não existindo diferença significativa entre colocação de cateter venoso central único ou múltiplo.

Discussão: Após implementação da estratégia multidimensional não se registou nos Cuidados Intensivos Pediátricos ocorrência de infeções da corrente sanguínea associadas ao uso de cateter venoso central.

Conclusões: Devem ser encetados esforços para preservar o mesmo grau de prevenção multidimensional, para que se confirme a redução efetiva da taxa de incidência de infeções da corrente sanguínea associadas ao uso de cateter venoso central.

Palavras-chave: Cateteres Venosos Centrais; Infecções Relacionadas a Cateter; Infecção Hospitalar; Unidades de Cuidados Intensivos Pediátricos.

\section{ABSTRACT}

Introduction: To determine the central-line associated bloodstream infection rate after implementation of central venous catheter-care practice bundles and guidelines and to compare it with the previous central-line associated bloodstream infection rate.

Material and Methods: A prospective, longitudinal, observational descriptive study with an exploratory component was performed in a Pediatric Intensive Care Unit during five months. The universe was composed of every child admitted to Pediatric Intensive Care Unit who inserted a central venous catheter. A comparative study with historical controls was performed to evaluate the result of the intervention (group 1 versus group 2).

Results: Seventy five children were included, with a median age of 23 months: 22 (29.3\%) newborns; 28 (37.3\%) with recent surgery and $32(43.8 \%)$ with underlying illness. A total of 105 central venous catheter were inserted, the majority a single central venous catheter $(69.3 \%)$, with a mean duration of $6.8 \pm 6.7$ days. The most common type of central venous catheter was the short-term, nontunneled central venous catheter $(45.7 \%)$, while the subclavian and brachial flexure veins were the most frequent insertion sites (both $25.7 \%)$. There were no cases of central-line associated bloodstream infection reported during this study. Comparing with historical controls (group 1), both groups were similar regarding age, gender, department of origin and place of central venous catheter insertion. In the current study (group 2), the median length of stay was higher, while the mean duration of central venous catheter (excluding peripherally inserted central line) was similar in both groups. There were no statistical differences regarding central venous catheter caliber and number of lumens. Fewer children admitted to Pediatric Intensive Care Unit had central venous catheter inserted in group 2, with no significant difference between single or multiple central venous catheter.

Discussion: After multidimensional strategy implementation there was no reported central-line associated bloodstream infection Conclusions: Efforts must be made to preserve the same degree of multidimensional prevention, in order to confirm the effective reduction of the central-line associated bloodstream infection rate and to allow its maintenance.

Keywords: Catheter-Related Infections; Central Venous Catheters; Cross Infection; Intensive Care Units, Pediatric.

1. Faculdade de Medicina. Universidade de Coimbra. Coimbra. Portugal.

2. Serviço de Cuidados Intensivos Pediátricos. Hospital Pediátrico. Centro Hospitalar e Universitário de Coimbra. Coimbra. Portugal.

3. Unidade de Neurodesenvolvimento e Autismo. Serviço do Centro de Desenvolvimento da Criança. Centro de Investigação e Formação Clínica. Hospital Pediátrico. Centro Hospitalar e Universitário de Coimbra. Coimbra. Portugal.

$\square$ Autor correspondente: Andrea Dias. sofia.andrea@gmail.com

Recebido: 24 de outubro de 2015 - Aceite: 21 de março de 2016| Copyright @ Ordem dos Médicos 2016 


\section{INTRODUÇÃO}

Um acesso vascular central seguro é um dos aspetos essenciais da prática clínica moderna, indispensável nos serviços de cuidados intensivos neonatais e pediátricos para suprir necessidades energéticas, hídricas e eletrolíticas, realizar colheitas sanguíneas e administrar terapêuticas, em doentes cujo estado clínico o exige. ${ }^{1-10}$ As infeções da corrente sanguínea associadas ao uso de cateter central (ICSACC) são um dos tipos mais frequentes de infeções associadas aos cuidados de saúde, resultando em prolongamento do internamento, elevada morbilidade e mortalidade e aumento dos custos hospitalares. ${ }^{1-5,8,10-12}$ Para que ocorra uma ICSACC é necessário que os microrganismos tenham acesso à superfície intraluminal ou extraluminal do cateter venoso central (CVC), com subsequente adesão e formação de biofilme, permitindo-lhes uma infeção sustentada com posterior disseminação hematogénea. ${ }^{1,12,19}$ Este acesso ocorre através de um de quatro mecanismos: migração da microflora cutânea do doente ao longo do trajeto subcutâneo, com colonização da ponta do CVC; contaminação do lúmen ou das conexões; colonização por via hematogénea; e, mais raramente, por contaminação da solução perfundida. ${ }^{1,12,19} \mathrm{O}$ mecanismo extraluminal surge mais frequentemente com dispositivos de curta duração, enquanto a colonização intraluminal é mais frequente em CVC de longa duração. ${ }^{1,12}$ Outros aspetos que podem contribuir para aparecimento de ICSACC são: tamanho, diâmetro, número de lumens, tipo e material de composição do CVC; local de inserção; técnica de higiene e assepsia; tempo de cateterização; frequência de manipulação; e virulência do agente infetante. ${ }^{1,12,19}$ Os microrganismos mais frequentemente envolvidos neste processo incluem Staphylococcus coagulase-negativo (epidermidis), Staphylococcus aureus e Escherichia coli. ${ }^{9}$

Perante a suspeita de ICSACC recomenda-se a retirada do CVC, com base na apreciação clínica individual. Preconiza-se antibioterapia sistémica; todavia, os antibióticos são muitas vezes incapazes de erradicar os microrganismos presentes no biofilme, pelo que têm vindo a ser utilizadas várias soluções para encerramento de CVC de modo a destruir os biofilmes com eficácia e segurança variáveis. ${ }^{9}$

$\mathrm{Na}$ última publicação do National Healthcare Safety Network (NHSN), a taxa estimada de ICSACC em unidades de cuidados intensivos pediátricos médico-cirúrgicos era de 1,4 infeções por 1000 dias de cateter, valor que tem decrescido progressivamente ao longo da última década, ${ }^{7,14} \mathrm{O}$ que se atribui à implementação de normas de boa prática e estratégias multidimensionais. ${ }^{7,13}$ As taxas de ICSACC variam consideravelmente conforme a dimensão do hospital, o tipo de serviço e de CVC utilizado. Estas taxas, adicionalmente, podem também ser influenciadas por fatores de risco intrínsecos aos doentes ${ }^{12}$. A maioria desta informação tem origem em estudos efetuados em adultos, pelo que se tivermos em conta a heterogeneidade e os fatores de risco particulares da população pediátrica, concluímos que são necessários mais estudos para conhecer e melhorar o im- pacto destas intervenções.

No Serviço de Cuidados Intensivos do Hospital Pediátrico, Centro Hospitalar e Universitário de Coimbra (CIPE-HP/ CHUC), a redução da taxa de ICSACC foi desde sempre um objetivo, reportando-se uma incidência de 1,7 infeções por 1000 dias de exposição a CVC no último estudo referente a esta temática, no período de 2010 a $2011 . .^{15} \mathrm{~A}$ introdução e o reforço de intervenções multidisciplinares de educação, monitorização e prevenção destas infeções são de importância reconhecida na otimização da prestação de serviços de saúde ${ }^{4,6}$. O objetivo deste trabalho foi o de realizar um estudo da incidência de episódios de ICSACC após a implementação de um conjunto de medidas multidisciplinares em comparação com controlos históricos. ${ }^{15}$

\section{MATERIAL E MÉTODOS}

O CIPE do HP-CHUC constitui um serviço de referência para a Região Centro de Portugal e integra-se num hospital altamente diferenciado, classificado em grupo III. Possui doze camas de internamento, incluindo cuidados intensivos e intermédios, disponibilizando cuidados médico-cirúrgicos a uma população de cerca de 450000 crianças e adolescentes com menos de 18 anos..$^{20}$ Interna cerca de 400 doentes por ano.

Conduziu-se no serviço um estudo observacional descritivo de vertente exploratória, longitudinal, com colheita de dados prospetiva, de 6 de setembro de 2013 a 5 de fevereiro de 2014 (cinco meses). O universo do estudo foi constituído pelas crianças admitidas no CIPE neste período, submetidas à colocação de um ou mais CVC ou com CVC in situ. Foram considerados os seguintes tipos de CVC: de curta duração, de longa duração tunelizado, de longa duração com reservatório, CVC de hemodiafiltração, cateter venoso umbilical (CVU), e cateter central de introdução periférica ou epicutâneo-cava (EPC).

Previamente ao estudo, foi efetuada pelos autores uma sessão de esclarecimento e formação aos profissionais de saúde abordando a política de colocação e manutenção de CVC e regras de prevenção de ICSACC. ${ }^{4,16-18}$ Procedeu-se na mesma sessão à apresentação de uma 'Ficha de Colocação de CVC' (Anexo 1 - http://www.actamedicaportuguesa.com/revista/index.php/amp/article/view/5558/373380_Apendice01.pdf) e de uma 'Ficha de Manutenção de CVC' (Anexo 2 - http://www.actamedicaportuguesa.com/ revista/index.php/amp/article/view/5558/ 373-380_Apendice02.pdf) adaptadas de Wheeler $\mathrm{D}$, et al. ${ }^{2}$

Foram avaliadas as seguintes variáveis demográficas: idade, género, proveniência, motivo de internamento, antecedentes patológicos, presença de infeção na admissão e duração de internamento. Foram colhidos os dados constantes da 'Ficha de Colocação' e na 'Ficha de Manutenção' do CVC.

$O$ protocolo de enfermagem do CIPE, relativo à manutenção de CVC, segue as diretrizes propostas pelo Centers for Disease Control and Prevention, National Healthcare Safety Network (CDC-NHSN) em relação à prevenção de 
ICSACC,${ }^{25}$ nomeadamente no que diz respeito à higiene das mãos e assepsia, mudança de penso e limpeza do local de inserção e substituição dos sistemas. A desinfeção do local de inserção é feita com iodopovidona ou álcool a $70 \%$. Em relação à substituição dos pensos, o tratamento do local de inserção é executado cada sete dias quando é utilizado adesivo, ou cada dois se for utilizado penso tipo compressa. A substituição dos sistemas depende das soluções perfundidas, sendo trocados cada 72 horas se forem soluções eletrolíticas ou outras medicações, cada 48 horas em caso de alimentação parenteral sem lípidos ou 24 horas se contiver lípidos ou perfusão isolada de lípidos, ou substituição imediata após administração de hemoderivados e citostáticos.

Para o diagnóstico das ICSACC, foram utilizadas as definições do CDC-NHSN. A ICSACC é definida como uma infeção da corrente sanguínea confirmada laboratorialmente (ICSCL) que ocorreu 48 horas após colocação e permanência de um CVC, na ausência de outro foco infecioso, na presença de pelo menos um dos critérios de ICSCL. ${ }^{21}$ Se o doente foi transferido de outro serviço ou instituição de saúde já com um CVC in situ, a ICSACC só foi considerada da responsabilidade do CIPE se surgiu 48 horas após a admissão. Nos doentes que tiveram alta com CVC in situ, verificou-se se surgiu ICSACC nas 48 horas após a alta.

Após a retirada do CVC, a ponta foi enviada sistematicamente para cultura. $\mathrm{O}$ isolamento de gérmen, na ausência de sintomas ou sinais clínicos de infeção, foi considerado colonização de cateter.

A taxa de ICSACC é calculada através da razão entre o número de ICSACC e o número de dias de CVC, multiplicado por $1000 .{ }^{26}$ A presença de pelo menos um CVC no doente é considerada como um dia de CVC, de acordo com as orientações do NHSN. ${ }^{22}$

Os resultados do estudo atual foram comparados com os obtidos em estudo prévio, entre 1 de julho e 31 de dezembro de 2010 (seis meses). ${ }^{15}$ Foram assim constituídos dois grupos: grupo 1 (controlos históricos) e grupo 2 (estudo atual).

Foi realizada a análise estatística dos dados recorrendo a IBM SPSS Statistics Software, versão 20. A caraterização dos grupos efetuou-se através da determinação de frequências absolutas e relativas para variáveis qualitativas e pelo cálculo de medidas de tendência central e de dispersão para variáveis quantitativas. Efetuou-se a análise comparativa (grupo 1 versus grupo 2) considerando-se um nível de significância de $p=0,05$, utilizando para comparar variáveis qualitativas nominais os testes Qui-quadrado e exato de Fisher e para comparar variáveis quantitativas e qualitativas ordinais ou intervalares o teste de Mann-Whitney e o teste $t$ de Student.

\section{RESULTADOS}

No período considerado, foram admitidos no CIPE 75 doentes com CVC (75/173; 43,4\%), 58,7\% do género masculino. A mediana de idade foi 23 meses $\left(P_{25} 4\right.$ dias; $P_{75}$ 12,5 anos) e $45,3 \%$ (34/75) encontravam-se no primeiro
Tabela 1 - Distribuição da amostra de acordo com faixa etária

\begin{tabular}{lc}
\hline & $\mathbf{n}(\%)$ \\
\hline$<28$ dias (recém-nascido) & $22(29,3)$ \\
IG 32 - 37 semanas & 9 \\
IG $<32$ semanas & 4 \\
$\mathbf{2 8}$ dias a 1 ano (lactente) & $12(16)$ \\
$\geq 1$ ano e < 12 anos (criança) & $19(25,3)$ \\
$\geq 12$ anos (adolescente) & $22(29,3)$ \\
Total & 75 \\
\hline
\end{tabular}

IG: Idade gestacional

ano de vida. Vinte e dois dos 75 doentes (29,3\%) eram recém-nascidos (com menos de 28 dias de vida); destes, treze $(59,1 \%)$ eram prematuros (Tabela 1$)$.

A mediana de duração de internamento no CIPE foi de sete dias $\left(P_{25}\right.$ dois dias; $P_{75} 15$ dias $)$, variando de um a 77 dias. O internamento hospitalar teve uma duração mediana de 19 dias $\left(P_{25}\right.$ nove dias; $P_{75} 36$ dias). Vinte e oito dos 75 doentes $(37,3 \%)$ foram submetidos a intervenção cirúrgica, dos quais quatro (14,3\%) eram RN. Quarenta e oito doentes (64\%) apresentavam patologia subjacente: 15 (20\%) eram doentes oncológicos, cinco $(6,7 \%)$ eram transplantados (quatro hepáticos e um de medula óssea) e 28 $(37,3 \%)$ sofriam de outras patologias. Três doentes $(4 \%)$ foram submetidos a hemodiafiltração, três (4\%) foram admitidos com queimaduras graves e 10 (13,3\%) realizaram nutrição parenteral.

A proveniência mais frequente foi do bloco operatório em 33 doentes (44\%), seguido de outras unidades hospitalares em 24 casos (32\%). Cinquenta e cinco doentes $(73,3 \%)$ foram admitidos no CIPE com CVC in situ. Relativamente à evolução, 50 doentes $(66,7 \%)$ tiveram alta para outros serviços mantendo o CVC in situ, enquanto em 25 (33,3\%) o CVC foi removido durante o internamento no CIPE. Procedeu-se a reinserção de novo CVC em 14 $(18,7 \%)$.

A mediana do Pediatric Index of Mortality II $\left(\mathrm{PIM}_{2}\right)$ foi de $2,4 \%$ para o subgrupo de doentes que colocou CVC $\left(P_{25}\right.$ 0,59\%; $\mathrm{P}_{75} 6,5 \%$ ). A mediana global do $\mathrm{PIM}_{2}$ no CIPE para o mesmo período foi de $0,95 \%\left(P_{25} 0,45 \% ; P_{75} 4,2 \%\right)$, enquanto a mortalidade global foi de quatro em $173(2,3 \%)$. Três doentes incluídos no estudo faleceram (4\%), nenhum deles por infeção associada aos cuidados de saúde.

Foram colocados, no total, 105 CVC nos 75 doentes incluídos, correspondente a 613 dias de cateter. Na maioria $(69,3 \%)$ só foi colocado um CVC; 23 crianças $(30,7 \%)$ necessitaram de colocação de um segundo cateter e sete $(9,3 \%)$ de um terceiro. O tipo de CVC mais frequentemente colocado foi o de curta duração $(48 / 105,45,7 \%)$, seguido do EPC (28/105, 26,7\%) (Tabela 2). Quando colocados mais do que um CVC $(30 / 105,28,6 \%)$, o tipo mais utilizado foi o EPC $(17 / 30,56,7 \%)$. Os locais preferenciais de colocação foram as veias da flexura braquial e a subclávia (27; $25,7 \%)$ seguindo-se a femoral $(25 ; 23,8 \%)$. 
Tabela 2 - Tipo e localização dos CVC utilizados

\begin{tabular}{|c|c|c|c|}
\hline & $\begin{array}{c}\text { CVC }_{1} \\
\mathrm{n}=75\end{array}$ & $\begin{array}{c}\mathrm{CVC}_{2} \\
\mathrm{n}=23\end{array}$ & $\begin{array}{l}\mathrm{CVC}_{3} \\
\mathrm{n}=7\end{array}$ \\
\hline \multicolumn{4}{|l|}{ Tipo } \\
\hline CVC de curta duração & $40(53,3 \%)$ & $7(30,4 \%)$ & $1(14,3 \%)$ \\
\hline CVU & $13(17,3 \%)$ & $3(13,0 \%)$ & 0 \\
\hline EPC & $11(14,7 \%)$ & $11(47,8 \%)$ & $6(85,7 \%)$ \\
\hline CVC de longa duração com reservatório & $6(8,0 \%)$ & $1(4,3 \%)$ & 0 \\
\hline CVC de hemodiafiltração & $4(5,3 \%)$ & 0 & 0 \\
\hline CVC de longa duração tunelizado & $1(1,3 \%)$ & $1(4,3 \%)$ & 0 \\
\hline \multicolumn{4}{|l|}{ Localização } \\
\hline Veia subclávia & $24(32,0 \%)$ & $3(13,0 \%)$ & 0 \\
\hline Veias da flexura braquial & $10(13,3 \%)$ & $11(47,8 \%)$ & $6(85,7 \%)$ \\
\hline Veia femoral & $20(26,7 \%)$ & $4(17,4 \%)$ & $1(14,3 \%)$ \\
\hline Veia umbilical & $13(17,3 \%)$ & $3(13,0 \%)$ & 0 \\
\hline Veia jugular interna & $7(9,3 \%)$ & $1(4,3 \%)$ & 0 \\
\hline Veia jugular externa & $1(1.3 \%)$ & 0 & 0 \\
\hline Veia safena & 0 & $1(4,3 \%)$ & 0 \\
\hline
\end{tabular}

EPC: Epicutâneo-cava; CVU: Cateter venoso umbilical; CVC: Cateter venoso central

Quando o CVC foi colocado no CIPE, a localização preferencial foi a veia femoral $(9 / 19 ; 47,4 \%)$, enquanto nos doentes que foram admitidos já com CVC in situ, o local mais frequente de colocação foi a veia subclávia (22/56; $39,3 \%$ ). O número mediano de lumens foi dois (1 a 3 lumens) e o diâmetro mediano foi $4 \mathrm{Fr}(4 \mathrm{a} 8 \mathrm{Fr}$ ). $\mathrm{O}$ tempo médio de permanência do CVC (média $\pm \mathrm{DP}$ ) foi 6,8 $\pm 6,7$ dias. Relativamente ao material de composição, 96 CVC $(91,4 \%)$ eram compostos de poliuretano e nove $(8,6 \%)$ de silicone.

A grande maioria dos CVC foi colocado por um médico especialista $(34,3 \%, 36 / 105)$. Os CVC foram mais frequentemente removidos por um profissional de enfermagem $(10 / 105,9,5 \%)$, seguido do médico especialista (8/105, 7,6\%) e do médico interno (7/105, 6,7\%); em 24,8\% (26/105) não foi possivel obter esta informação. A maioria das crianças $(54 / 105,51,4 \%)$ teve alta do CIPE com CVC in situ. O tempo mediano até à colocação completa do CVC foi de cinco minutos ( $P_{25} 5$ minutos; $P_{75} 10$ minutos). Naqueles que fizeram colheitas pelo CVC $(39 / 75,52 \%)$, fizeram-no em mediana três vezes $\left(P_{25} 1 ; P_{75} 6\right)$.

Foram cumpridas as normas de inserção de acordo com a 'Ficha de Colocação do CVC' no respeitante à higiene das mãos, manutenção de assepsia, máxima barreira de proteção assegurada e preparação adequada do material previamente à inserção em $100 \%$ dos CVC colocados no CIPE. Foi colocado apenas um CVC de forma ecoguiada, na jugular interna. O desinfetante cutâneo usado foi a iodopovidona em $100 \%$ dos casos. Nenhum dos CVC foi colocado em situação de emergência. Refere-se ainda que 55 do total de CVC incluídos no estudo foram inseridos pre- viamente à admissão no CIPE, não podendo ser confirmado quem colocou o CVC e o cumprimento das normas de inserção.

Foram cumpridas as normas de boas práticas estabelecidas na 'Ficha de Manutenção do CVC', nomeadamente discussão diária da necessidade de vias, sua integridade e cumprimento do previsto nas guidelines relativamente à manutenção e manipulação, em todos os CVC incluídos no estudo.

Os fluidos mais frequentemente perfundidos foram os solutos de glicose $(89,3 \%)$ e os cristaloides $(98,6 \%)$ (Tabela 3). A 41 doentes foi administrada perfusão de hemoderivados $(54,7 \%)$, sendo o mais frequente o concentrado de glóbulos vermelhos $(21,3 \%)$. Foi realizada antibioterapia em 58 (77,3\%) doentes. A antibioterapia foi instituída quer por motivo de internamento de foro infecioso, quer por profilaxia antibiótica pré e pós-operatória, quer por complicações e intercorrências infeciosas durante o internamento. Em nenhum caso se iniciou antibioterapia por suspeita de ICSACC.

Não se verificaram infeções no local de inserção do CVC. No período de estudo, não ocorreram ICSACC. Verificou-se colonização da ponta do CVC em 12 casos, sendo o Staphylococcus epidermidis o microrganismo mais frequentemente isolado. Oito doentes apresentavam infeção na admissão no CIPE (quatro infeções respiratórias, um infeção urinária, um sépsis e dois ICSACC). O início destas ICSACC precedeu o internamento no CIPE não sendo, por isso, contabilizadas.

Comparando o grupo de doentes incluídos no estudo atual (grupo 2) com o grupo de doentes que colocou CVC 
Tabela 3 - Perfusões administradas através do CVC

\begin{tabular}{lcc}
\hline & $\mathbf{n = 7 5 ( \% )}$ & $\begin{array}{c}\text { Duração em dias } \\
\left.\text { (mediana, } \mathbf{P}_{25} \text { e } \mathbf{P}_{75}\right)\end{array}$ \\
\hline Cristaloides & $74(98,7)$ & $4\left(\mathrm{P}_{25} 2 ; \mathrm{P}_{75} 11\right)$ \\
Solutos de glicose & $67(89,3)$ & $4\left(\mathrm{P}_{25} 4 ; \mathrm{P}_{75} 10\right)$ \\
Antibióticos & $58(77,3)$ & $6\left(\mathrm{P}_{25} 2 ; \mathrm{P}_{75} 10\right)$ \\
Hemoderivados & $41(54,7)$ & $2\left(\mathrm{P}_{25} 1 ; \mathrm{P}_{75} 4\right)$ \\
Inotrópicos & $25(33,3)$ & $3\left(\mathrm{P}_{25} 1,5 ; \mathrm{P}_{75} 6,5\right)$ \\
Aminoácidos & $24(32,0)$ & $5\left(\mathrm{P}_{25} 4 ; \mathrm{P}_{75} 7,75\right)$ \\
Lípidos & $23(30,7)$ & $6\left(\mathrm{P}_{25} 4 ; \mathrm{P}_{75} 9\right)$ \\
Bicarbonato & $10(13,3)$ & $1\left(\mathrm{P}_{25} 1 ; \mathrm{P}_{75} 6\right)$ \\
Propofol & $3(4,0)$ & $5\left(\mathrm{P}_{25} 3 ; \mathrm{P}_{75}-\right)$ \\
\hline
\end{tabular}

nos controlos históricos (grupo 1$)^{15}$ (Tabela 4), não se verificou diferença significativa em relação à mediana de idade (15,9 meses no grupo 1 vs 23 meses no grupo 2; Mann-Whitney $U$ test, $p=0,787$ ), ao género (Chi-Square, $p=$ $0,178$ ), à proveniência (Chi-Square, $p=0,05)$ e à gravidade da situação clínica à admissão (mediana $\mathrm{PIM}_{2}$ 1,34\% no grupo 1 vs 2,4\% no Grupo 2; Mann-Whitney $U$ test, $p=$ 0,351).

A duração mediana de internamento foi superior no grupo 2 (quatro dias no grupo 1 versus sete dias no grupo 2; Mann-Whitney $U$ test, $p=0,03$ ), sendo colocado CVC numa menor percentagem de crianças internadas no grupo 2 (69\% no grupo 1 vs 43,4\% no grupo 2; Chi-Square, $p<0,001)$. Não verificámos diferenças significativas entre os dois grupos em relação à percentagem de crianças que colocou CVC único ou múltiplo (Chi-Square, $p=0,06$ ) e em relação ao local de colocação do CVC (Chi-Square, $p=$ 0,05).

Considerando todos os tipos de CVC, e os EPC isoladamente, o número médio de dias de permanência, em ambas as situações foi superior no grupo 2 (4,1 $\pm 3,5$ dias no grupo 1 vs 6,8 $\pm 6,7$ dias no Grupo 2, teste $t$ Student $p<$ 0,001 para todos os tipos de CVC; e 5,1 $\pm 4,5$ dias no grupo 1 vs 11,8 $\pm 9,1$ dias no grupo 2, teste $t$ Student $p=0,001$ para os EPC). Considerando o tempo médio de permanência de CVC excluindo o EPC, não se verificou diferença significativa entre os dois grupos (3,95 \pm 4,04 dias no grupo 1 vs 4,97 \pm 4,25 dias no grupo 2; teste t Student $p=0,075$ ). Não se verificaram diferenças significativas relativamente ao número de lumens do CVC - excluindo EPC, CVU e CVC de longa duração com reservatório - (Mann-Whitney $\mathrm{U}$ test, $p=0,282$ ) e ao calibre - excluindo EPC e CVC de longa duração com reservatório - (Mann-Whitney U test, $p=0,079$ ) em ambos os grupos.

\section{DISCUSSÃO}

As ICSACC estão associadas a aumento significativo da morbilidade e mortalidade, nomeadamente nos serviços de cuidados intensivos pediátricos, onde a maioria dos doentes possuem condições clínicas que inspiram grandes cuidados e a necessidade de acessos vasculares centrais é muitas das vezes imprescindível. ${ }^{1-11}$

No período em estudo, após as intervenções estabelecidas, não se reportaram ICSACC no internamento do CIPE do HP-CHUC. Este é um indício positivo, que suporta uma possível correlação entre as intervenções aplicadas e a redução da incidência de ICSACC. Para reforçar esta conclusão fez-se uma análise comparativa das características demográficas das duas populações. Comparando os dados do presente trabalho com a população de controlos históricos, não existiu diferença significativa entre os dois grupos em relação ao género, à idade, à proveniência dos doentes e à gravidade da situação clínica na admissão, apresentando características semelhantes e portanto passíveis de comparação. Há, contudo, a referir a existência de duas ICSACC, resultantes de internamento prévio à admissão no CIPE. Não existe uma associação clara entre os fatores de risco apresentados por estas duas crianças e o aparecimento de ICSACC na população pediátrica, embora alguns estudos reportem que a idade inferior a dois anos e a presença de patologia oncológica possam constituir fatores de risco. ${ }^{9,23,24}$

Verificou-se colonização da ponta do CVC em 12 casos Apesar das recomendações atuais (2016) não recomendarem a cultura da ponta do $\mathrm{CVC}^{28}$ (ao contrário do indicado nas guidelines mais recentes à data do estudo, de 2011), ${ }^{21}$ a sua realização permitiu conhecer os principais microrganismos responsáveis pela colonização dos cateteres no serviço, bem como o seu perfil de sensibilidade aos antimicrobianos.

Não obstante a ausência de ICSACC, a população estudada apresentava várias condições que poderiam estar associadas a um risco aumentado de infeção. São considerados fatores de risco para ICSACC na população pediátrica: a idade inferior a dois anos (o que corresponde a quase metade da população estudada, sendo que cerca de um terço eram recém-nascidos); baixo peso (< $8 \mathrm{~kg})$; transplante de células estaminais hematopoiéticas (o que ocorreu num paciente); antecedentes recentes de cirurgia e patologia crónica associada (presentes em cerca de um terço da população estudada); exposição a procedimentos invasivos além da colocação de CVC - como o uso de ventilação mecânica, a entubação, a colocação de cateter arterial, colocação de dreno torácico ou a nutrição por via parentérica (necessários em cerca de $20 \%$ da população); a permanência prolongada de CVC e o uso de múltiplos CVC, o que sucedeu em cerca de um terço dos doentes deste estudo. 9,25

Verificou-se neste grupo de estudo uma duração mediana de internamento superior à dos controlos históricos, resultante sobretudo do fato de ambos os grupos representarem amostras pequenas, bastando um número pequeno de casos admitidos com tempo de internamento mais prolongado, como ocorreu no grupo 2, para alterar significativamente a duração de internamento da população total. Apesar do aumento da duração de internamento estar diretamente relacionado com o risco de surgimento 
Tabela 4 - Caraterísticas da população em estudo e da população dos controlos históricos

\begin{tabular}{|c|c|c|c|}
\hline & Grupo 2 & Grupo $1^{15}$ & $p^{\text {a }}$ \\
\hline Duração do estudo & $\begin{array}{c}5 \text { meses } \\
\text { (set 2013- fev 2014) }\end{array}$ & $\begin{array}{c}6 \text { meses } \\
\text { (jul - dez 2011) }\end{array}$ & \\
\hline $\begin{array}{l}\text { Doentes com CVC }(n / \%) \\
\text { Total de admissões }(n)\end{array}$ & $\begin{array}{c}75(43,4 \%) \\
173\end{array}$ & $\begin{array}{c}117(69 \%) \\
169\end{array}$ & $<0,001$ \\
\hline $\begin{array}{l}\text { Idade (meses) } \\
\text { Mediana } \\
\mathrm{P}_{25} \text { e } \mathrm{P}_{75} \\
\text { Mínimo e máximo }\end{array}$ & $\begin{array}{c}23 \\
0 ; 150 \\
0,03-218\end{array}$ & $\begin{array}{c}15,9 \\
2 ; 111,6 \\
0,03-211,5\end{array}$ & 0,787 \\
\hline $\begin{array}{l}\text { Género }(\mathrm{n} / \%) \\
\text { Masculino } \\
\text { Feminino }\end{array}$ & $\begin{array}{l}44(58,7 \%) \\
31(41,3 \%)\end{array}$ & $\begin{array}{l}57(48,7 \%) \\
60(51,3 \%)\end{array}$ & 0,178 \\
\hline $\begin{array}{l}\text { Duração de Internamento (dias) } \\
\text { Mediana } \\
\mathrm{P}_{25} \text { e } \mathrm{P}_{75} \\
\text { Mínimo e máximo }\end{array}$ & $\begin{array}{c}7 \\
2 ; 15 \\
1-77\end{array}$ & $\begin{array}{c}4 \\
2 ; 7,5 \\
1-37\end{array}$ & 0,03 \\
\hline $\begin{array}{l}\text { Proveniência (n / \%) } \\
\text { Bloco Operatório } \\
\text { Outro Hospital } \\
\text { Serviço de Urgência/UICD } \\
\text { Serviço de Medicina } \\
\text { Serviços de Cirurgia }\end{array}$ & $\begin{array}{c}33(44 \%) \\
24(32 \%) \\
13(17,3 \%) \\
4(5,4 \%) \\
1(1,3 \%)\end{array}$ & $\begin{array}{c}43(36,8 \%) \\
46(39,3 \%) \\
8(6,8 \%) \\
17(14,5 \%) \\
3(2,6 \%)\end{array}$ & 0,05 \\
\hline $\begin{array}{l}\mathrm{PIM}_{2}(\%) \\
\text { Mediana } \\
\mathrm{P}_{25} \text { e } \mathrm{P}_{75} \\
\text { Mínimo e máximo }\end{array}$ & $\begin{array}{c}2,4 \\
0,59 ; 6,5 \\
0,13-69,67\end{array}$ & $\begin{array}{c}1,34 \\
0,8 ; 4,7 \\
0,13-99,22\end{array}$ & 0,351 \\
\hline Mortalidade & $3(4,0 \%)$ & $2(1,7 \%)$ & \\
\hline $\begin{array}{l}\text { Número de CVC (n / \%) } \\
\text { CVC único } \\
\text { Múltiplos CVC }\end{array}$ & $\begin{array}{l}52 / 75(69,3 \%) \\
23 / 75(30,7 \%)\end{array}$ & $\begin{array}{l}95 / 117(81,2 \%) \\
22 / 117(18,8 \%)\end{array}$ & 0,06 \\
\hline $\begin{array}{l}\text { Duração do CVC (dias) } \\
\text { Média } \pm \text { DP } \\
\text { Mínimo e máximo }\end{array}$ & $\begin{array}{c}6,79 \pm 6,7 \\
{[1-42]}\end{array}$ & $\begin{array}{c}4,1 \pm 3,5 \\
{[1-24]}\end{array}$ & $<0,001$ \\
\hline Calibre (mediana, mínimo e máximo; Fr) ${ }^{\mathrm{b}}$ & $4[4-8]$ & $4[3-5,5]$ & 0,079 \\
\hline Lúmen (mediana, mínimo e máximo; $\left.\mathrm{n}^{\circ}\right)^{\mathrm{c}}$ & $2[1-3]$ & $2[1-3]$ & 0,282 \\
\hline $\begin{array}{l}\text { Localização (n) } \\
\text { Veia subclávia } \\
\text { Veia femoral } \\
\text { Veia umbilical } \\
\text { Veias jugulares }\end{array}$ & $\begin{array}{c}27 \\
25 \\
16 \\
9\end{array}$ & $\begin{array}{c}49 \\
48 \\
9 \\
11\end{array}$ & 0,05 \\
\hline $\begin{array}{l}\text { EPC } \\
\text { Número de EPC colocados } \\
\text { Duração (média } \pm \text { DP, em dias) }\end{array}$ & $\begin{array}{c}26 \\
11,82 \pm 9,12\end{array}$ & $\begin{array}{c}27 \\
5,12 \pm 4,45\end{array}$ & 0,001 \\
\hline ICSACC (n / taxa, infeções por dias de exposição a cateter) & 0 & $1(1,7)$ & 0,644 \\
\hline Dias de exposição a CVC (dias) & 613 & 592 & \\
\hline
\end{tabular}

CVC: Cateter venoso central; UICD: Unidade de Internamento de Curta Duração; EPC: Epicutâneo-cava; DP: Desvio padrão.

${ }^{a}$ Comparação efetuada por teste $t$ de Student e Mann-Whitney para variáveis quantitativas e qualitativas ordinais ou intervalares e Chi-Square para variáveis qualitativas nominais. ${ }^{b}$ Os cálculos envolvendo o número de lumens contabilizam todos os CVC, exceto os EPC e os CVC de longa duração com reservatório.

'Os cálculos envolvendo o calibre contabilizam todos os CVC, exceto os EPC, os CVC de longa duração com reservatório e os cateteres venosos umbilicais (CVU).

de ICSACC, ${ }^{23}$ tal não se verificou. A média do tempo de permanência dos CVC foi maior no presente estudo, o que pode constituir também fator de risco. ${ }^{6,24,25}$ Este facto deveu-se, sobretudo, à maior duração de permanência dos
EPC. Os EPC são dispositivos recomendados para uso de longa duração, com menor risco infecioso que os de curta duração; contudo a permanência para além de trinta e cinco dias aumenta esse risco. ${ }^{6,19}$ Excluindo os EPC, o tempo 
de permanência dos CVC foi inferior no presente estudo, podendo contribuir para a redução da taxa de infeção.

O material de composição da quase totalidade dos CVC era poliuretano, em conformidade com as mais recentes normas de boas práticas. ${ }^{19,23,25}$

Não se verificou redução do número de lumens do CVC, relativamente ao grupo de controlos históricos. Na literatura atual, não se encontrou relação causal entre a ocorrência de ICSACC e o número mais elevado de lumens do CVC à exceção dos CVC de longa duração tunelizados; no entanto, faz parte das diretrizes de boas práticas o uso de menor número de lumens possível. ${ }^{19,23,25}$

A implementação do preenchimento da 'Ficha de colocação de CVC' e da 'Ficha de manutenção de CVC' de forma sistemática, com alto nível de compliance na maioria dos casos, obrigou a maior cuidado diário relativo aos CVC, contribuindo assim para a melhoria dos cuidados prestados pelo CIPE. Segundo relatos prévios, a introdução deste conjunto de intervenções, padronizando os processos de colocação, manutenção e remoção de CVC e procurando avaliar diariamente, junto dos profissionais de saúde, a necessidade e integridade das vias, demonstrou uma redução significativa na incidência de ICSACC. . 2,3,9-11,13,15,16-19,25,27 $^{2}$

Há, contudo, que salientar algumas limitações deste trabalho. Primeiro, o processo de colheita de dados foi influenciado pela colaboração dos profissionais no preenchimento dos formulários das tarefas diárias, podendo por vezes ter ocorrido falha na colheita de dados em tempo oportuno e assim conduzir a alguma interferência na precisão nos resultados finais. Adicionalmente, uma vez que foi impossível verificar individualmente o cumprimento das normas na colocação, manutenção e remoção de cada CVC, o estudo baseou-se nos dados reportados por quem colocou o CVC, podendo ser alvo de viés.

Secundariamente, o estudo restringiu-se apenas a um serviço - o CIPE - que é um serviço hospitalar pediátrico especializado, pelo que os valores aqui obtidos poderão não ser passíveis de extrapolação para outras unidades hospitalares, inclusive serviços de cuidados intensivos não pediátricos.

Em terceiro lugar, algumas das recomendações referidas nas guidelines mais recentes do CDC para a prevenção de ICSACC não puderam ser cumpridas, nomeadamente no que diz respeito à preferência pelo uso de solução de clorhexidina para desinfeção cutânea prévia à inserção do CVC, à colocação ecoguiada do CVC e à preferência pela inserção do CVC na veia subclávia em detrimento da femoral. ${ }^{19} \mathrm{~A}$ solução de clorhexidina a $2 \%$ não se encontrava disponível no período de estudo, sendo alternativamente utilizadas soluções de etanol a $70 \%$ ou iodopovidona. Apenas alguns estudos reportam associação estabelecida entre o uso de soluções de clorhexidina e a diminuição da incidência de ICSACC, pelo que nas recomendações atuais não são dadas indicações precisas para o uso de qualquer uma dessas substâncias. ${ }^{11,13,19}$ Comparando a população admitida com CVC in situ e a população que colocou CVC durante internamento no CIPE, verificou-se a preferência pela localização femoral na colocação de CVC no CIPE, resultado da maior experiência da inserção neste local, apesar de contrário às recomendações que sugerem que se reduza a utilização desta via, dando preferência à veia subclávia. ${ }^{19}$ São pontos a reequacionar em futuras intervenções.

Finalmente, apesar dos resultados conseguidos, como não se verificaram ICSACC, não foi possível, como era objetivo inicial deste trabalho, determinar possíveis fatores de risco ou protetores para ICSACC na população em estudo. Não obstante estes factos, esta investigação contribuiu para aumentar o nosso nível de conhecimento sobre o tema, permitindo-nos contribuir para melhores cuidados nesta área. Será recomendável a realização de futuras monitorizações da eficácia destas medidas, para confirmação da diminuição efetiva dos casos de ICSACC e avaliação do efeito e do benefício mantido das intervenções multidisciplinares.

\section{CONCLUSÃO}

A redução da ICSACC e, em maior grau, de infeções associadas aos cuidados de saúde, deve ser considerada como um dos principais objetivos de qualquer serviço hospitalar, uma vez que são passíveis de prevenção na sua quase totalidade. ${ }^{1-11}$

No CIPE, ao longo destes cinco meses, não se registou a ocorrência de qualquer ICSACC, o que nos aponta para a redução da taxa de incidência atual de ICSACC do CIPE tendo em conta a percentagem de 1,7 infeções por 1000 dias de exposição a CVC no estudo previamente efetuado neste serviço. ${ }^{15}$ Apesar de se tratar de um período de estudo relativamente curto, as diretrizes de boa prática e intervenções multidisciplinares introduzidas evidenciam um claro impacto positivo, corroborando os indícios suportados por estudos semelhantes realizados em outras unidades hospitalares a nível internacional.

Os resultados obtidos permitem afirmar a importância deste tipo de estratégia na prevenção de infeções hospitalares, sendo de recomendar a sua implementação de forma sistemática a nível nacional.

Torna-se fundamental reunir esforços a nível hospitalar e mais particularmente dos serviços para manter elevados níveis de exigência na prestação de cuidados, nomeadamente através de protocolos atualizados, de forma a confirmar a efetiva redução da taxa de ICSACC a médio e longo prazo. O registo sistemático das taxas de infeção associada aos cuidados de saúde e a sua análise de forma regular em plataformas pré-definidas, de que são exemplo as plataformas disponibilizadas pela DGS (http://www.insa-rios.net/), são de incentivar.

\section{AGRADECIMENTOS}

Os autores agradecem a disponibilidade e imprescindível cooperação de todos os profissionais do Serviço de Cuidados Intensivos Pediátricos do Hospital Pediátrico de Coimbra - nomeadamente equipa médica, equipa de enfermagem e equipa administrativa - na elaboração deste trabalho. 


\section{PROTECÇÃO DE PESSOAS E ANIMAIS:}

Os autores declaram que os procedimentos seguidos estavam de acordo com os regulamentos estabelecidos pelos responsáveis da Comissão de Investigação Clínica e Ética e de acordo com a Declaração de Helsínquia da Associação Médica Mundial.

\section{CONFIDENCIALIDADE DOS DADOS:}

Os autores declaram ter seguido os protocolos do seu centro de trabalho acerca da publicação de dados.

\section{REFERÊNCIAS}

1. Safdar N, Maki D. The pathogenesis of catheter-related bloodstream infection with noncuffed short-term central venous catheters. Intensive Care Med. 2004;30:62-7.

2. Wheeler D, Giaccone MJ, Hutchinson N, Haygood M, Bondurant P, Demmel $\mathrm{K}$ et al. A hospital-wide quality improvement collaborative to reduce catheter-associated bloodstream infections. Pediatrics. 2011;128;e995.

3. Provonost P, Needham D, Berenholtz S, Sinopoli D, Chu H, Cosgrove $S$ et al. An intervention to decrease catheter-related bloodstream infections in the ICU. N Eng J Med. 2006;355:2725-32.

4. Exline M, Ali NA, Zikri N, Mangino JE, Torrence K, Vermillion B et al. Beyond the bundle - journey of a tertiary care medical intensive care unit to zero central-line associated bloodstream infections. Critical Care. 2013;17:R41.

5. Niedner MD, Huskins C, Colantuoni E, Muschelli J, Harris M, Rice T et al. Epidemiology of central line-associated bloodstream infections in the Pediatric Intensive Care Unit. Infec Control Hosp Epidemiol. 2011;32:1200-8.

6. Almeida A, Pereira O, Neto MT, Casella P. Cateteres vasculares centrais no recém-nascido: recomendações para prevenção de infeção relacionada com ou associada a cateteres vasculares centrais. Lisboa: Sociedade Portuguesa de Neonatologia; 2012.

7. Edwards JR, Peterson KD, Banerhee S, Allen-Bridson K, Morrell G, Dudeck MA et al. National Healthcare Safety Network report: Data summary for 2006 through 2008. Am J Infect Control. 2009;37:783-805.

8. Miller M, Niedner MF, Huskins WC, Colantuoni E, Yenokvan G, Moss $\mathrm{M}$ et al. Reducing PICU central line-associated bloodstream infections: 3-year results. Pediatrics. 2011;128:1077-83.

9. Janum S, Zingg W, Afshari A, Classen V. Bench-to-bedside review: challenges of diagnosis, care and prevention of central catheter-related bloodstream infections in children. Critical Care. 2013;17:238.

10. Marra A, Rodrigues RG, Durão MS, Correa L, Guastelli LR, Faria Moura D et al. Impact of a program to prevent central line-associated bloodstream infection in the zero tolerance era. Am J Infect Control. 2010;38:434-9.

11. Hatler C, Buckwald L, Salas-Allison Z, Murphy-Taylor C. Evaluating central venous catheter care in a pediatric intensive care unit. Am J Crit Care. 2009;18:514-20.

12. Pina E, Silva EG, Costa VM, Neto T, Estrada J, Estrada H. et al. Recomendações para a prevenção da infeção nosocomial associada aos dispositivos intravasculares. Lisboa: Plano Nacional para o Controlo de Infeção; 2006.

13. Galpern D, Guerrero A, Tu A, Fahoum B, Wise, L. Effectiveness of a central line bundle campaign on line-associated infections in the intensive care unit. Surgery. 2008;144:492-5.

14. Dudeck MA, Weiner LM, Allen-Bridson K, Malpiedi PJ, Peterson KD, Pollock DA et al. National Healthcare Safety Network report: data summary for 2012, device-associated module. Am J Infect Control.

\section{CONFLITOS DE INTERESSE}

Os autores declaram não terem qualquer conflito de interesse relativamente ao presente artigo.

\section{FONTES DE FINANCIAMENTO}

Os autores declaram não ter recebido subsídios ou bolsas para a elaboração do artigo.

2013;41:1148-66.

15. Caldeira M. Infeções da Corrente Sanguínea Associadas aos Cuidados de Saúde numa Unidade de Cuidados Intensivos Pediátricos [trabalho final de mestrado]. Coimbra: Faculdade de Medicina da Universidade de Coimbra; 2011.

16. Band JD, Gaynes R. Prevention of intravascular catheter-related infections. UpToDate. 2013. [consultado 2014 mar 30] Disponível em: http://www.uptodate.com/home

17. Li S, Faustino EV, Golombek S. Reducing central line infections in pediatric and neonatal patients. Curr Infect Dis Resp. 2013;15:269-77.

18. Li S, Bizzarro MJ. Prevention of central line associated bloodstream infections in critical care units. Curr Opin Pediatr. 2011;23:85-90.

19. Centers for Disease Control and Prevention. Guidelines for the Prevention of Intravascular Catheter-Related Infections, 2011. [consultado 2014 mar 30]. Disponível em: http://www.cdc.gov/hicpac/ pdf/guidelines/bsi-guidelines-2011.pdf.

20. Instituto Nacional de Estatística, I.P. - Censos 2011, Resultados definitivos - Portugal. Lisboa: INE; 2012.

21. Centers for Disease Control and Prevention. Central line-associated bloodstream infection (CLABSI) event. [consultado 2014 mar 30]. Disponível em: http://www.cdc.gov/nhsn/pscmanual/4psc clabscurrent. pdf.

22. Horan TC, Andrus M, Dudeck MA. CDC/NHSN surveillance definition of health care-associated infection and criteria for specific types of infections in the acute care setting. Am J Infect Control. 2008;36:309-32.

23. Yogaraj JS, Elward AM, Fraser VJ. Rate, risk factors and outcomes of nosocomial primary bloodstream infection in Pediatric Intensive Care Unit patients. Pediatrics. 2002;110:481-5.

24. Urrea M, Iriondo M, Thio M, Krauel X, Serra M, LaTorre C, et al. A prospective incidence study of nosocomial infections in a neonatal care unit. Am J Infect Control. 2003;31:505-7.

25. Odetola FO, Moller FW, Dechert RE, Van der Elzen K, Chenoweth C. Nosocomial catheter-related bloodstream infections in a pediatric intensive care unit: risks and rates associated with various intravascular technologies. Pediatr Crit Care Med. 2003;4:432-6.

26. Warren DK, Zack JE, Elward AM, Cox MJ, Fraser VJ. Nosocomial primary bloodstream infections in intensive care unit patients in a nonteaching community medical center: a 21-month prospective study. Clin Infect Dis. 2001;33:1329-35.

27. Berenholtz SM, Provonost PJ, Lipsett PA, Hobson D, Earsing K, Farley JE et al. Eliminating catheter-related bloodstream infections in the intensive care unit. Crit Care Med. 2004;32:2014-20.

28. Centers for Disease Control and Prevention. Bloodstream Infection Event (Central Line-Associated Bloodstream Infection and NonCentral Line-Associated Bloodstream Infection), 2016. [consultado a 2016 fev 26]. Disponível em: http:// http://www.cdc.gov/nhsn/pdfs/ pscmanual/4psc_clabscurrent.pdf. 